\title{
Variants of acinar adenocarcinoma of the prostate mimicking benign conditions
}

\author{
Peter A Humphrey \\ Department of Pathology, Yale School of Medicine, New Haven, CT, USA
}

\begin{abstract}
Histological variants of acinar adenocarcinoma of the prostate may be of significance due to difficulty in diagnosis or due to differences in prognosis compared to usual acinar adenocarcinoma. The 2016 World Health Organization classification of acinar adenocarcinoma includes four variants that are deceptively benign in histological appearance, such that a misdiagnosis of a benign condition may be made. These four variants are atrophic pattern adenocarcinoma, pseudohyperplastic adenocarcinoma, microcystic adenocarcinoma, and foamy gland adenocarcinoma. They differ from usual small acinar adenocarcinoma in architectural glandular structure and/or cytoplasmic and nuclear alterations. The variants are often admixed, in variable proportions, with usual small acinar adenocarcinoma that is often Gleason pattern 3 but may be high-grade pattern 4 in a minority of cases. Atrophic pattern adenocarcinoma can be identified in a sporadic setting or after radiation or hormonal therapy. This variant is characterized by cytoplasmic volume loss and can resemble benign glandular atrophy, an extremely common benign process in the prostate. The glands of pseudohyperplastic adenocarcinoma simulate usual epithelial hyperplasia, with gland complexity that is not typical of small acinar adenocarcinoma. These complex growth configurations include papillary infoldings, luminal undulations, and branching. Microcystic adenocarcinoma is characterized by cystic dilation of prostatic glands to a size that is much more commonly observed in cystic change in benign prostatic glands. Finally, the cells in foamy gland adenocarcinoma display cytoplasmic vacuolization and nuclear pyknosis, features that can found in benign glands and macrophages. Three of the four variants (atrophic, pseudohyperplastic, and microcystic) are assigned low-grade Gleason pattern 3. Of significance, foamy gland adenocarcinoma can be Gleason pattern 3 but can also be high-grade pattern 4 or 5 . Diagnostic awareness of the existence of these deceptively benignappearing variants of acinar adenocarcinoma is essential so that an accurate diagnosis of prostate cancer may be rendered.
\end{abstract}

Modern Pathology (2018) 31, S64-S70; doi:10.1038/modpathol.2017.137

Adenocarcinoma of the prostate may be deceptively benign-appearing upon light microscopic examination of hematoxylin-and-eosin-stained tissue sections. These 'pseudobenign' carcinomas may be missed in needle biopsy tissue and contribute to the false negative rate of about $1-3 \%$ in needle biopsy diagnosis of prostate cancer. ${ }^{1-3}$ The basis for a misdiagnosis of adenocarcinoma of the prostate in needle core tissue as benign includes a relatively low number of glands, intermingling with benign glands, lack of disorganization, and the presence of variants of prostate cancer that may mimic benign conditions. $^{1,2}$

Variants of usual acinar adenocarcinoma are, according to the 2016 World Health Organization

Correspondence: Professor PA Humphrey, MD, PhD, Department of Pathology, Yale School of Medicine, Box 208023, 310 Cedar Street, New Haven, CT 06520, USA.

E-mail: peter.humphrey@yale.edu

Received 22 July 2017; accepted 17 August 2017 scheme, the following: atrophic; pseudohyperplastic; microcystic; foamy; mucinous (colloid); signet ringlike cell; pleomorphic giant cell; and sarcomatoid (Table 1). ${ }^{4}$ The first four variants-atrophic, pseudohyperplastic, microcystic, and foamy adenocarcinoma-may morphologically mimic benign processes in the prostate and are the focus of this review.

\section{Atrophic pattern adenocarcinoma}

Atrophic pattern adenocarcinoma may be seen in a sporadic setting or following radiation or androgen deprivation therapy and is characterized by cytoplasmic volume loss, just like benign atrophy. ${ }^{5-8}$ In comparison, usual acinar adenocarcinoma exhibits a moderate amount of cytoplasm. The following discussion relates to sporadic atrophic pattern adenocarcinoma. The incidence of sporadic atrophic pattern adenocarcinoma is $2 \%$ in needle core cases ${ }^{6}$ 
Table 12016 WHO classification of histological variants of acinar adenocarcinoma of the prostate

Atrophic

Pseudohyperplastic

Microcystic

Foamy gland

Mucinous (colloid)

Signet ring-like cell

Pleomorphic giant cell

Sarcomatoid

and $16 \%$ in radical prostatectomy cases. ${ }^{7}$ It has also been reported in two transurethral resection of prostate cases. ${ }^{5}$ There are no reported gross descriptions of atrophic pattern adenocarcinoma. Microscopically, an infiltrative arrangement of glands displaying cytoplasmic volume loss (Figure 1), macronucleoli, and nucleomegaly, along with admixed non-atrophic usual acinar adenocarcinoma with a moderate amount of cytoplasm, is typical. The percentage of the tumor that is comprised of glands with atrophic features is variable. On average, about $16-25 \%$ of the total adenocarcinoma harbors glands with atrophic features. ${ }^{6,7}$ The range is broad, being $10-90 \%$ in needle core cases ${ }^{6}$ and $1-70 \%$ in radical prostatectomy cases. ${ }^{6,7}$ The atrophic malignant glands are often intermingled with malignant glands with the usual moderate amount of cytoplasm (Figure 2). Note that none of the reported cases were pure atrophic pattern adenocarcinoma. Pure atrophic pattern adenocarcinoma should rarely, if ever, be diagnosed, particularly in needle biopsy tissue, due to diagnostic difficulty in recognition and distinction from benign atrophy (see below). The glands are often rounded to oval and typically small (Figures 1 and 2) but cystically dilated glands of microcystic adenocarcinoma may also be lined by carcinoma cells with cytoplasmic volume loss, that is, with atrophic features ${ }^{6,7,9}$ Challenges in diagnosis include identification, in some glands, of nuclear atypia, due to nuclear compression (Figure 2), and diminished expression of AMACR with $70 \%$ of cases being positive, ${ }^{10}$ compared to $~ 90 \%$ of cases of usual acinar adenocarcinoma. ${ }^{11}$ Complete loss of basal cells in all atrophic pattern adenocarcinoma glands is seen in immunohistochemistry using antibodies directed against high-molecular-weight cytokeratins (such as antibody $34 \beta E 12$ ) and p63. ${ }^{7}$

The differential diagnosis of atrophic pattern adenocarcinoma vs benign atrophy is critical. Infiltrative growth, nuclear atypia, and complete and diffuse absence of basal cells are the most reliable criteria for diagnosis of atrophic pattern adenocarcinoma rather than benign atrophy. ${ }^{5-7}$ Intraluminal pink secretions, intraluminal wispy blue mucin, and intraluminal crystalloids achieve statistical significance in being found more often in atrophic pattern adenocarcinoma than benign atrophy, ${ }^{7}$ but are detected in only a minority of atrophic pattern

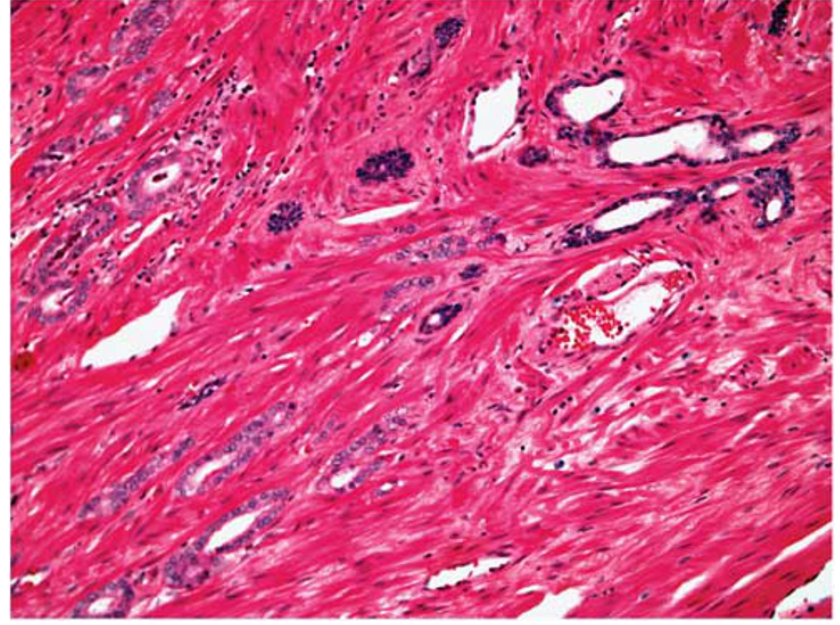

Figure 1 Atrophic pattern adenocarcinoma (lower left side) with infiltrative glands with cytoplasmic volume loss, compared to benign atrophy (upper right side).

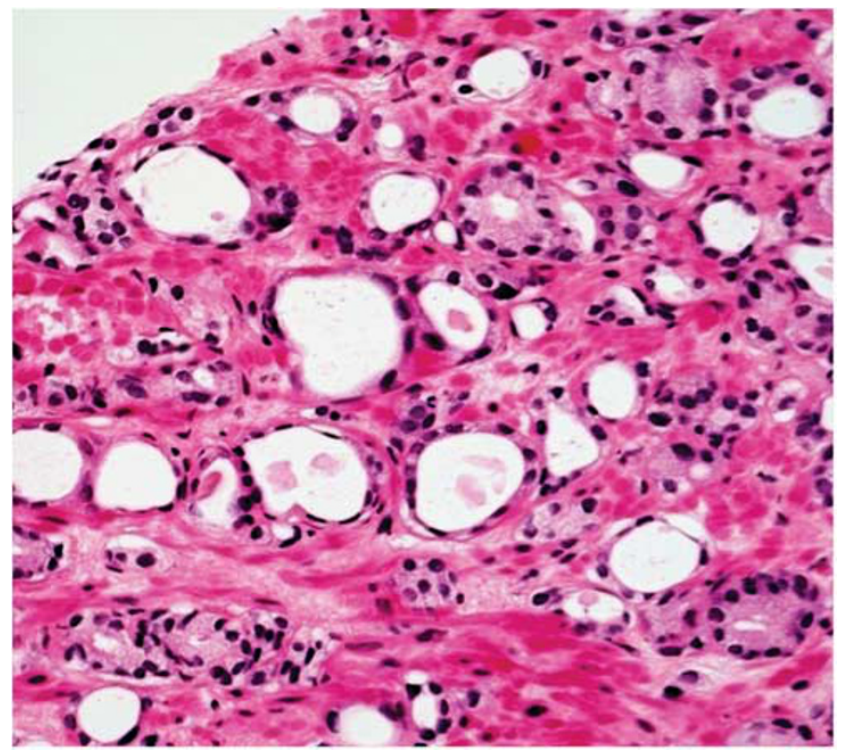

Figure 2 Atrophic pattern adenocarcinoma admixed with usual small acinar adenocarcinoma glands with a moderate amount of cytoplasm.

adenocarcinoma cases, which limits diagnostic utility. Perineural invasion by atrophic pattern adenocarcinoma may also be identified in a minority of cases. ${ }^{7}$ There is immunophenotypic overlap that can confound the differential diagnosis of atrophic pattern adenocarcinoma vs benign atrophy. Scattered benign atrophic glands are negative for basal cell immunostains in $6-23 \%$ of cases. ${ }^{12-17}$ AMACR can be positive in $4 \%$ of cases of benign atrophy, ${ }^{11,18}$ and positive in $10-79 \%$ of partial atrophy cases. ${ }^{12,13,16}$ Thus, benign atrophy may display a cancer-like immunophenotype with AMACR overexpression and basal cell loss. Benign atrophy is negative for ERG expression ${ }^{19}$ but only around $50 \%$ of prostatic adenocarcinomas are positive for ERG, 
such that a negative result has limited diagnostic value.

Atrophic pattern adenocarcinoma is a proliferative Gleason pattern 3 adenocarcinoma. Of note, sporadic atrophic pattern adenocarcinomas are not quiescent or dying glands. The proliferation index of atrophic pattern adenocarcinoma (at $4 \%$ ) is similar to usual acinar adenocarcinoma (at $5 \%$ ) and apoptosis is rare. ${ }^{7}$ Most atrophic pattern adenocarcinomas are Gleason pattern 3 and the associated usual acinar adenocarcinoma is usually pattern 3 , although in $13 \%$ of cases high-grade pattern 4 is present in the usual acinar component. ${ }^{7}$ The presence of atrophic features in adenocarcinoma is not likely of prognostic significance as adenocarcinomas with and without atrophic change do not differ in Gleason grade or pathologic stage. ${ }^{7}$

\section{Pseudohyperplastic adenocarcinoma}

Pseudohyperplastic adenocarcinoma can simulate the appearance of usual epithelial hyperplasia. ${ }^{20-24}$ This variant accounts for a large percentage of needle biopsy cases misdiagnosed as benign., ${ }^{1,2}$ The incidence in adenocarcinoma needle core cases is $2 \%,{ }^{20}$ in chips from transurethral resection $3 \%,{ }^{23}$ and in the whole gland $11 \% .{ }^{20}$ There are no known clinical, radiological, or gross features specific for pseudohyperplastic adenocarcinoma. Microscopically, pseudohyperplastic adenocarcinoma displays papillary infoldings, luminal undulations, branching, and cystic dilatation (Figures 3 and 4). An infiltrative pattern can be detected in a minority of cases. ${ }^{20}$ This variant may be detected in the whole prostate gland in the peripheral or transition zones. In radical prostatectomy cases there is almost always associated usual small acinar adenocarcinoma, although in needle core tissues up to $90 \%$ of the adenocarcinoma may be pseudohyperplastic. ${ }^{21}$ The nuclei are

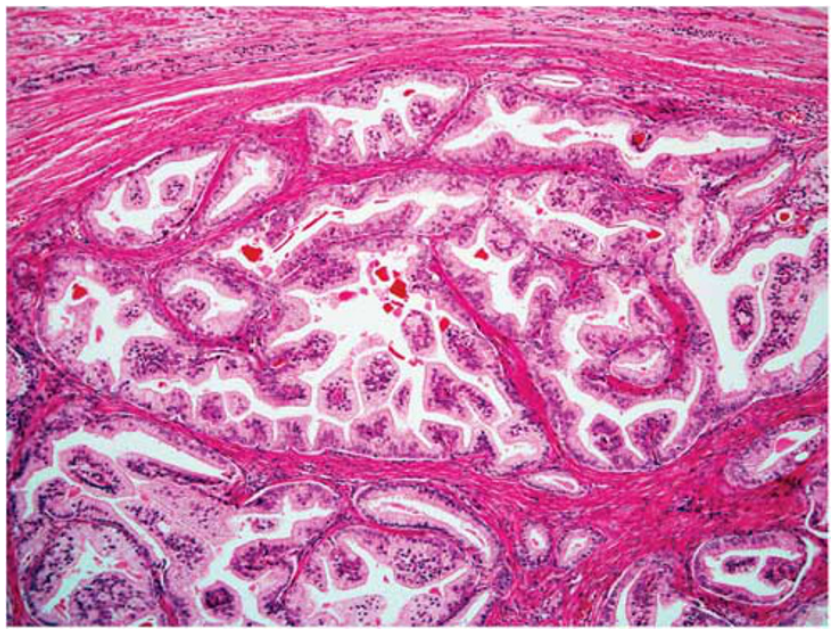

Figure 3 Pseudohyperplastic adenocarcinoma with papillae, intraluminal projections, and intraluminal crystalloids.
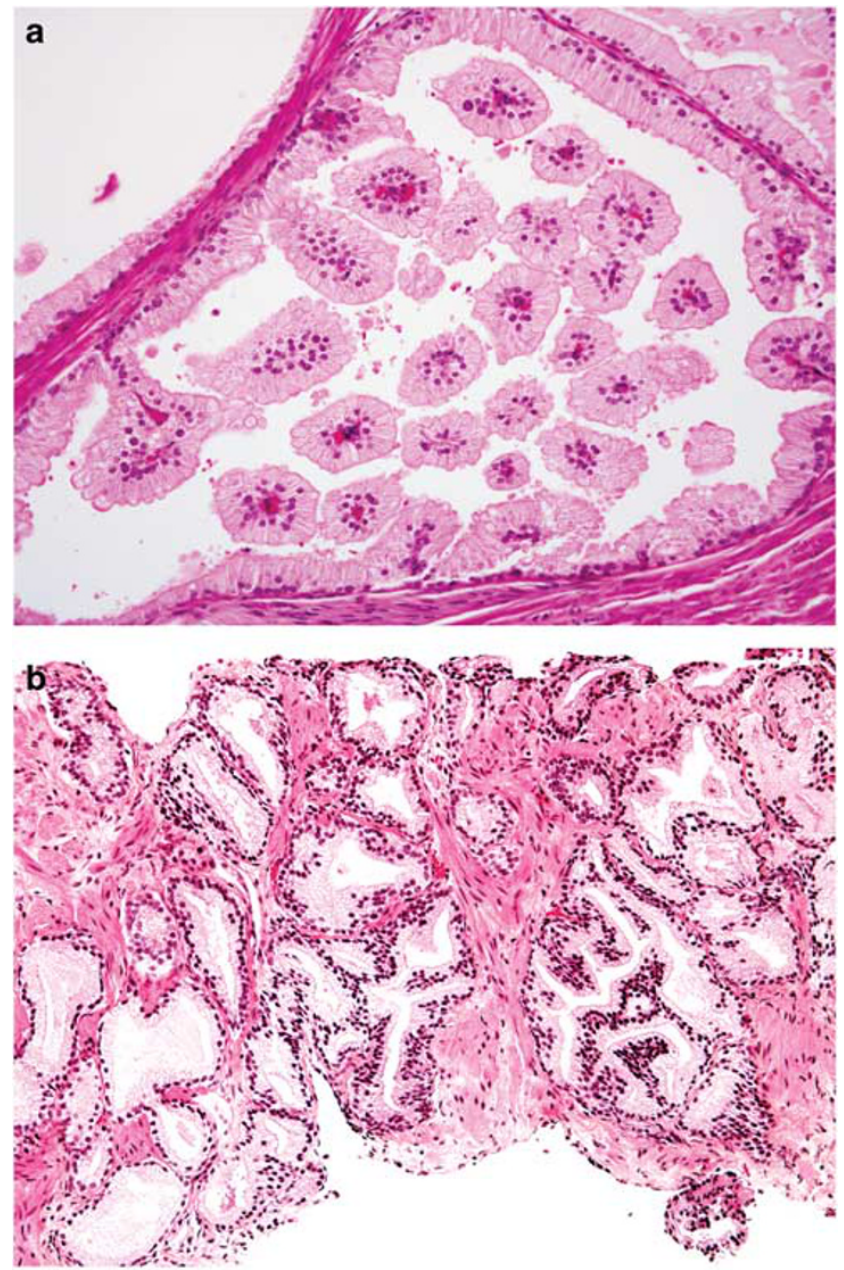

C

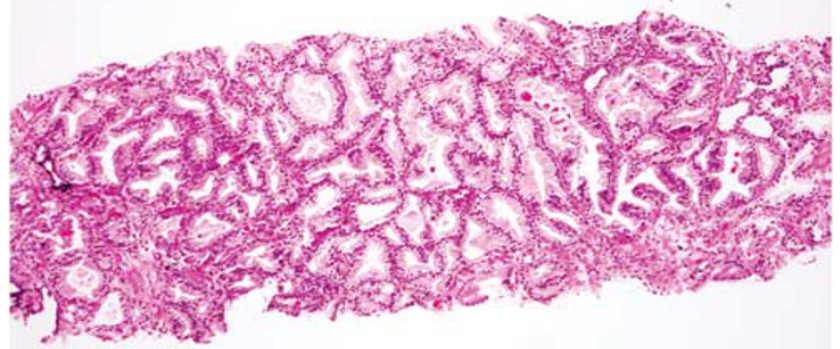

Figure 4 Pseudohyperplastic adenocarcinoma with papillary growth (a). Prostatic adenocarcinoma with pseudohyperplastic features in needle core tissue $(\mathbf{b}, \mathbf{c})$.

rounded and typically have prominent nucleoli although nuclear atypia may be minimal in some cases, particularly those uncommon cases with foamy cytoplasmic features. ${ }^{25}$ Features associated with prostatic neoplasia, such as intraluminal crystalloids, intraluminal pink amorphous secretions, 
and intraluminal wispy blue mucin, are seen in a minority of cases. ${ }^{20,21}$ Discovery of intraluminal crystalloids at low magnification in hyperplasticappearing glands (Figure 3) may provide a diagnostic clue as to the presence of pseudohyperplastic carcinoma.

The differential diagnosis centers on crowded benign glands with normal glandular architectural complexity, usual glandular hyperplasia of the prostate, and ductal adenocarcinoma. Findings that are useful in establishing a diagnosis of malignancy include gland crowding, nuclear enlargement, macronucleoli, and transition to usual small acinar adenocarcinoma. Search for adjacent typical small acinar adenocarcinoma is essential, and just as for atrophic pattern adenocarcinoma, a diagnosis of pure pseudohyperplastic adenocarcinoma should rarely, if ever, be rendered, especially in needle core tissue. There should be a low threshold for performance of immunohistochemistry in cases that are concerning for pseudohyperplastic adenocarcinoma, particularly in needle core tissue. AMACR expression is detectable in $70-83 \%$ of cases $^{26}$ and no basal cells are found in all the glands of concern, by immunostaining. Identification of perineural invasion by pseudohyperplastic glands can be diagnostically useful, but is observed in only a minority of cases. $^{20-22,27}$ The papillary growth of pseudohyperplastic adenocarcinoma may be mistaken not only for the papillae of usual prostatic epithelial hyperplasia $^{28}$ but also for the papillae of ductal adenocarcinoma of the prostate. The neoplastic epithelial lining of the fibrovascular cores of ductal adenocarcinoma papillary structures typically is pseudostratified with elongated nuclei. ${ }^{29}$ In contrast, the lining cells of pseudohyperplastic adenocarcinoma papillae are not pseudostratified and the nuclei are rounded and basal in location (Figure 4a). Distinction of papillary pseudohyperplastic adenocarcinoma from papillary ductal adenocarcinoma is important as the former is Gleason pattern 3 while the latter is high-grade pattern 4 .

Pseudohyperplastic adenocarcinomas are Gleason pattern 3 in the prostate, ${ }^{30}$ although pseudohyperplastic features have been reported in metastatic prostatic adenocarcinoma. ${ }^{20}$ The prognosis for patients with pseudohyperplastic prostatic adenocarcinoma is likely similar to that of Gleason pattern 3 usual small acinar adenocarcinoma. ${ }^{20}$ Of interest, HOXB13 G84E-related familial prostate cancers commonly show pseudohyperplastic features. ${ }^{31}$

\section{Microcystic adenocarcinoma}

Microcystic acinar adenocarcinoma exhibits gland dilatation with intermediate-sized glands that are, on average, 10 times the size of usual small acinar adenocarcinoma glands. ${ }^{9}$ That such dilated malignant glands exist in the prostate challenges the concept that prostatic adenocarcinoma is

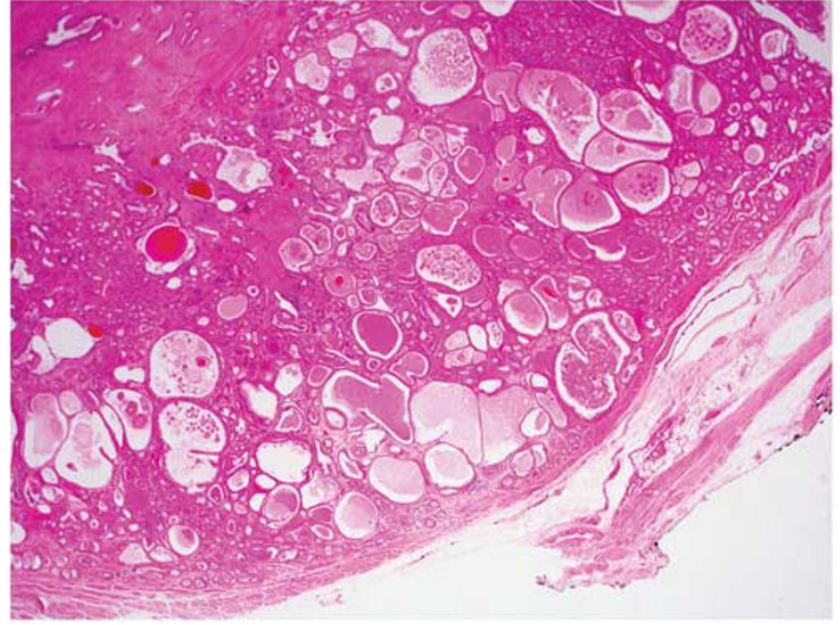

Figure 5 Microcystic adenocarcinoma: dilated malignant glands in a background of small acinar adenocarcinoma.

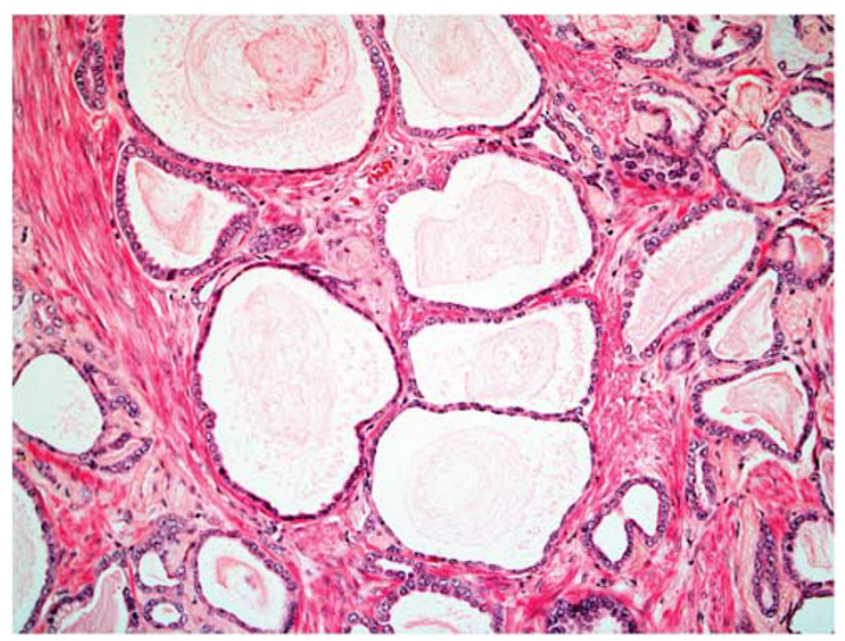

Figure 6 Microcystic adenocarcinoma with cystic glandular dilatation and atrophic features with cytoplasmic volume loss.

prototypically a small gland cancer. The incidence in radical prostatectomy cases is $11 \% .{ }^{9}$ Microscopically, the expansion of the luminal spaces generates a rounded profile, and the luminal cell lining layer is flat, with or without atrophic changes (Figures 5 and 6). Intraluminal crystalloids and blue mucin are uniformly present. There is always admixture with usual small acinar adenocarcinoma, which most often comprises the majority of the tumor. ${ }^{9}$ In immunohistochemistry the dilated glands lack basal cells and almost all cases (96\%) express AMACR. ${ }^{9}$

The differential diagnosis centers mainly on cystic hyperplasia in the transition zone and cystic atrophy in the peripheral zone. ${ }^{32}$ Detection of nuclear atypia, AMACR overexpression, lack of basal cells, and adjacent small acinar adenocarcinoma are useful clues in establishing a diagnosis of malignancy for these microcystic glands. Cystic dilatation of 
malignant prostatic glands can also be seen in ductal adenocarcinoma, where the cystic change can be microscopic or macroscopic. ${ }^{33,34}$ Microscopic cystic ductal adenocarcinomas have been termed cystadenocarcinomas. ${ }^{34}$ These cystically dilated ductal adenocarcinomas differ from microcystic acinar adenocarcinomas in that the cysts are larger (at $5-10 \mathrm{~mm}$ ) and exhibit intracystic papillae. ${ }^{34} \mathrm{In}$ contrast, the size of microcystic acinar adenocarcinoma glands ranges from 0.4 to $0.9 \mathrm{~mm}$ and the malignant lining layer is typically flat. ${ }^{9}$

The Gleason pattern assignment is 3 for microcystic adenocarcinoma. While this is a low-grade adenocarcinoma, in $36 \%$ of cases in the whole prostate there is pattern 4 elsewhere, ${ }^{9}$ indicating that detection of microcystic adenocarcinoma in prostate needle biopsy tissue does not guarantee that high-grade pattern 4 is not present elsewhere, and perhaps unsampled, in the whole gland. In uncommon cases $(n=2 \text { in } 1 \text { series of } 53 \text { cases })^{9}$ microcystic adenocarcinoma glands invade into periprostatic adipose tissue.

\section{Foamy gland adenocarcinoma}

Foamy gland adenocarcinoma can be deceptively benign-appearing due to lack of nuclear atypia. ${ }^{35-39}$ Clinically, the mean age at diagnosis is 65 years (range 50-78 years) ${ }^{36}$ and, like many men with pseudobenign carcinomas, these men present with an elevated serum prostate-specific antigen level. ${ }^{37}$ Microscopically, foamy gland adenocarcinoma is characterized by abundant foamy or xanthomatoustype cytoplasm with pyknotic nuclei being frequent (Figure 7). ${ }^{35-39}$ Intraluminal dense pink secretions are often identified. ${ }^{36}$ Foamy glands are observed admixed with usual acinar adenocarcinoma in 17\% of needle biopsy cases $^{38}$ and $13-23 \%$ of radical prostatectomy cases, ${ }^{39}$ and are only rarely found in pure form. The lack of nuclear atypia in the foamy glands can create difficulty in establishment of a malignant diagnosis, especially in needle core tissue. Unlike other deceptively benign-appearing prostatic adenocarcinomas, high Gleason grade foamy gland adenocarcinomas exist (Figure 8). Most foamy gland carcinomas are Gleason score 6 or 7 , although Gleason score 8-10 carcinomas may also be diagnosed. In immunohistochemistry AMACR overexpression has been reported in 68 (ref. 26)-92\% (ref. 39) of invasive foamy gland adenocarcinomas and basal cells are absent. ERG expression is found in $42 \%$ of cases but in most cases does not provide added diagnostic value beyond detection of AMACR. ${ }^{38}$ The foamy appearance of the cytoplasm is due to the presence of numerous intracytoplasmic vesicles, which lack lipid or neutral mucin. ${ }^{37}$

The differential diagnosis includes benign glands, which can have foamy cytoplasm, ${ }^{40}$ and foamy macrophages, including those seen in prostatic xanthomas $^{41,42}$ and granulomatous prostatitis,

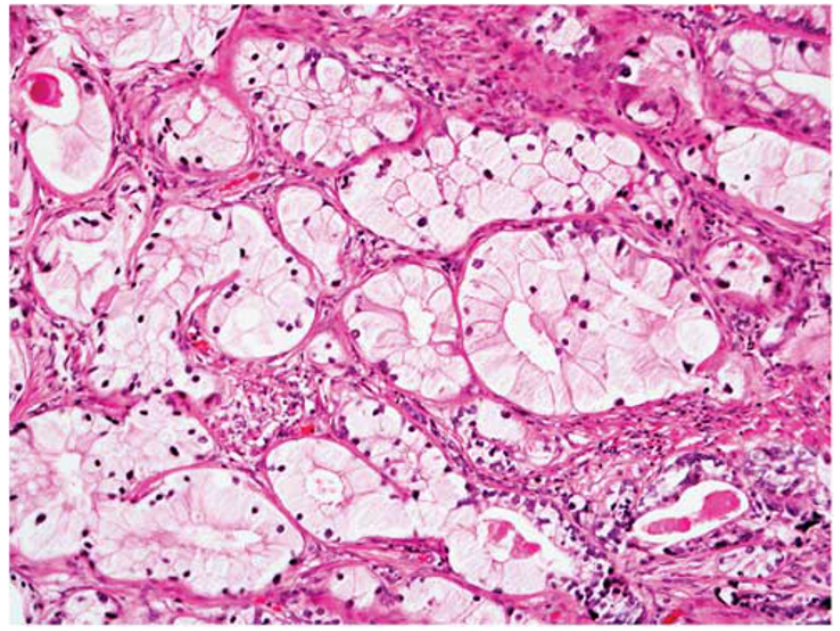

Figure 7 Foamy gland carcinoma: foamy cytoplasm and nuclear pyknosis.

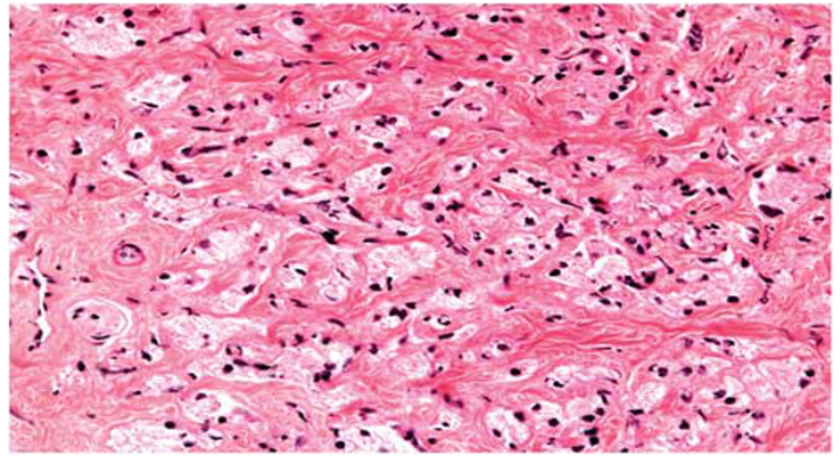

Figure 8 Foamy gland carcinoma: Gleason pattern 5.

especially when epithelioid histiocytes possess cleared to vacuolated cytoplasm (due to lipid accumulation). ${ }^{43,44}$ An immunohistochemical marker panel can be of diagnostic utility: positive reactions for pan-cytokeratin, PSA, PAP, and NKX3.1, with negative immunostains for CD68 and lysozyme, can be of aid in confirmation of foamy gland carcinoma when foamy histiocytes are a consideration. ${ }^{45,46}$ Neoplastic glands are also in the differential diagnosis: there are rare reported cases of foamy gland high-grade prostatic intraepithelial neoplasia ${ }^{47}$ and intraductal foamy gland carcinoma. ${ }^{36}$ Foamy change has also been described in a few cases of ductal adenocarcinoma of the prostate. $^{48}$ Finally, androgen deprivation therapy can induce cytological changes in prostatic adenocarcinoma cells that have an appearance similar to foamy gland carcinoma, with cytoplasmic vacuolization and nuclear pyknosis. ${ }^{49}$

Foamy gland carcinoma carries a similar prognosis as non-foamy gland adenocarcinoma after radical prostatectomy. ${ }^{39}$

\section{Disclosure/conflict of interest}

The author declares no conflict of interest. 


\section{References}

1 Carswell BM, Woda BA, Wang X, et al. Detection of prostate cancer by $\alpha$-methylacyl CoA racemase (P504S) in needle biopsy specimens previously reported as negative for malignancy. Histopathology 2006;48: 668-673.

2 Wolters T, van der Kwast TH, Vissers CJ, et al. Falsenegative prostate needle biopsies: frequency, histopathologic features, and follow-up. Am J Surg Pathol 2010;34:35-43.

3 Bonkoff H. Significance of prostate cancer missed on needle biopsy tools for retrieving missed cancer. Prostate 2016;76:369-375.

4 Humphrey PA, Amin MB, Berney DM et al. Acinar adenocarcinoma. In: Moch $\mathrm{H}$, Humphrey PA, Ulbright TM (eds). WHO Classification of Tumours of the Urinary System and Male Genital Organs, 4th edn. International Agency for Research on Cancer: Lyon, France, 2016, pp 138-161.

5 Cina SJ, Epstein JI. Adenocarcinoma of the prostate with atrophic features. Am J Surg Pathol 1997;21: 287-295.

6 Egan JM, Lopez-Beltran A, Bostwick DG. Prostatic adenocarcinoma with atrophic features: malignancy mimicking a benign process. Am J Surg Pathol 1997;21: 931-935.

7 Kaleem Z, Swanson PE, Vollmer RT, et al. Prostatic adenocarcinoma with atrophic features: a study of 202 consecutive, completely-embedded radical prostatectomy specimens. Am J Clin Pathol 1998;109:695-703.

8 Reuter VE. Pathological changes in benign and malignant tissue following androgen deprivation therapy. Urology 1997;49:16-22.

9 Yaskiv O, Cao D, Humphrey PA. Microcystic adenocarcinoma of the prostate: a variant of pseudohyperplastic and atrophic patterns. Am J Surg Pathol 2010;34:556-561.

10 Farinola MA, Epstein JI. Utility of immunohistochemistry for alpha-methylacyl-CoA racemase in distinguishing atrophic prostate cancer from benign atrophy. Hum Pathol 2004;35:1272-1278.

11 Hameed O, Humphrey PA. Immunohistochemistry in diagnostic surgical pathology of the prostate. Semin Diagn Pathol 2005;22:84-104.

12 Wang W, Sun X, Epstein JI. Partial atrophy on needle biopsy cases: a morphologic and immunohistochemical study. Am J Surg Pathol 2008;32:851-857.

13 Przbycin CG, Kunju LP, Wu AJ, et al. Partial atrophy in prostate needle biopsies; a detailed analysis of its morphology, immunophenotype, and cellular kinetics. Am J Surg Pathol 2008;32:58-64.

14 Amin MB, Tamboli P, Varma M, et al. Post-atrophic hyperplasia of the prostate gland: a detailed analysis of its morphology in needle biopsy specimens. Am J Surg Pathol 1999;23:925-931.

15 Hedrick L, Epstein JI. Use of keratin 903 as an adjunct in the diagnosis of prostate carcinoma. Am J Surg Pathol 1989;13:389-396.

16 Adley BP, Yang XJ. Alpha-methylacyl Coenzyme A racemase immunoreactivity in partial atrophy of the prostate. Am J Clin Pathol 2006;126:849-855.

17 Brimo F, Epstein JI. Immunohistochemical pitfalls in prostate pathology. Hum Pathol 2012;43:313-324.

18 Hameed O, Sublett J, Humphrey PA. Immunohistochemical stains for p63 and alpha-methylacyl-CoA racemase versus a cocktail comprising both, in the diagnosis of prostatic carcinoma: a comparison on the immunohistochemical staining of 430 foci in radical prostatectomy and needle biopsy tissues. Am J Surg Pathol 2005;29:579-587.

19 Yaskiv O, Zhang X, Simmerman K, et al. The utility of ERG/p63 double immunohistochemical staining in the diagnosis of limited cancer in prostate needle biopsies. Am J Surg Pathol 2011;35:1062-1068.

20 Humphrey PA, Kaleem Z, Swanson PE, et al. Pseudohyperplastic prostatic adenocarcinoma. Am J Surg Pathol 1998;22:1239-1246.

21 Wolf AN, Epstein JI. Pseudohyperplastic prostatic adenocarcinoma in needle biopsy and simple prostatectomy. Am J Surg Pathol 2000;24:1039-1046.

22 Arista-Nasr J, Martinez-Benitez B, Aguilar-Ayala EL, et al. Pseudohyperplastic prostate carcinoma: histologic patterns and differential diagnosis. Ann Diagn Pathol 2015;19:253-260.

23 Arista-Nasr J, Martiniez-Benitez B, Valdes S, et al. Pseudohyperplastic prostatic adenocarcinoma in transurethral resections of the prostate. Pathol Oncol Res 2003;9:232-235.

24 Humphrey PA. Pseudohyperplastic adenocarcinoma of the prostate. J Urol 2014;191:1416-1417.

25 Arista-Nasr J, Barranon-Martinez I, Aquilar-Ayala E, et al. Pseudohyperplastic adenocarcinoma with foamy changes in needle prostate biopsy and prostatectomy. Int J Surg Pathol 2016;24:477-482.

26 Zhou M, Jiang Z, Epstein JI. Expression and diagnostic utility of alpha-methylacyl-CoA-racemase (P504S) in foamy gland and pseudohyperplastic prostate cancer. Am J Surg Pathol 2003;27:772-778.

27 So JS, Epstein JI. Histologic features of pseudohyperplastic perineural invasion in prostatic adenocarcinoma: a mimicker of benign hyperplastic glands and high-grade prostatic intraepithelial neoplasia. Am J Surg Pathol 2014;38:852-857.

28 Humphrey PA, Young RH. Pseudoneoplastic lesions of the prostate. In: Humphrey PA, Manivel JC, Young RH (eds). Neoplastic Mimics in Genitourinary Pathology, Chapter 4, Figs 4.87 and 4.88. Demos Medical: New York, NY, USA, 2014, pp 140-142.

29 Egevad L, Epstein JI, Hameed O et al. Ductal adenocarcinoma. In: Moch H, Humphrey PA, Ulbright TM (eds). WHO Classification of Tumours of the Urinary System and Male Genital Organs. International Society for Research on Cancer: Lyon, France, 2016, pp 166-167.

30 Epstein JI, Allsbrook WC Jr, Amin MB, et al. The 2005 International Society of Urological Pathology (ISUP) consensus conference on Gleason grading of prostatic carcinoma. Am J Surg Pathol 2005;29:1228-1242.

31 Smith SC, Palanisamy N, Zuhlke KA, et al. HOXB13 G84E-related familial prostate cancers: a clinical, histologic, and molecular survey. Am J Surg Pathol 2014;38:615-626.

32 Humphrey PA, Young RH. Pseudoneoplastic lesions of the prostate. In: Humphrey PA, Manivel JC, Young RH (eds). Neoplastic Mimics in Genitourinary Pathology, Chapter 4, Fig. 4.39. Demos Medical: New York, NY, USA, 2014, p 113.

33 Kojima F, Kuike H, Matsuzaki I, et al. Macrocystic ductal adenocarcinoma of prostate: a rare gross appearance of prostate cancer. Ann Diagn Pathol 2017;27: 7-13.

34 Paner GP, Lopez-Beltran A, So JS, et al. Spectrum of cystic epithelial tumors of the prostate. Am J Surg Pathol 2016;40:886-895. 
35 Koca SB, Yildiz P, Behzatoglu K. Foamy gland carcinoma in core needle biopsies of the prostate: clinicopathologic and immunohistochemical study of 56 cases. Ann Diagn Pathol 2014;18:271-274.

36 Nelson RS, Epstein JI. 1996. Prostatic carcinoma with abundant xanthomatous cytoplasm. Foamy gland carcinoma. Am J Surg Pathol 1996;20:419-426.

37 Tran TT, Sengupta E, Yang XY. Prostatic foamy gland carcinoma with aggressive behavior. Histologic, immunohistochemical, and ultrastructural analysis. Am J Surg Pathol 2001;25:618-623.

38 Warrick JI, Humphrey PA. Foamy gland carcinoma of the prostate in needle biopsy: incidence, Gleason grade, and comparative alpha-methylacyl-CoA racemase versus ERG expression. Am J Surg Pathol 2013;37: 1709-1714.

39 Hudson J, Cao D, Vollmer R, et al. Foamy gland carcinoma of the prostate: incidence, Gleason grade, and early clinical outcome. Hum Pathol 2012;43: 974-979.

40 Humphrey PA, Young RH. Pseudoneoplastic lesions of the prostate. In: Humphrey PA, Manivel JC, Young RH (eds). Neoplastic Mimics in Genitourinary Pathology, Chapter 4, Fig. 4.42. Demos Medical: New York, NY, USA, 2014, p 115.
41 Sebo TJ, Bostwick DG, Farrow GM, et al. Prostatic xanthoma: a mimic of prostatic adenocarcinoma. Hum Pathol 1994;25:386-389.

42 Chuang AY, Epstein JI. Xanthoma of the prostate: a mimicker of high-grade prostatic adenocarcinoma. Am J Surg Pathol 2007;31:1225-1230.

43 Miekos E, Wlodarczyk W, Szram S. Xanthogranulomatous prostatitis. Int Urol Nephrol 1986;18:433-437.

44 Fox H. Nodular histiocytic prostatitis. J Urol 1966;96: 372-374.

45 Presti B, Weidner N. Granulomatous prostatitis and poorly differentiated prostate carcinoma. Their distinction with use of immunohistochemical methods. Am J Clin Pathol 1991;95:330-334.

46 Oppenheimer JR, Kahane H, Epstein JI. Granulomatous prostatitis on needle biopsy. Arch Pathol Lab Med 1997;121:724-729.

47 Berman DM, Yang X, Epstein JI. Foamy gland highgrade prostatic intraepithelial neoplasia. Am J Surg Pathol 2000;24:140-144.

48 Lee TK, Miller JS, Epstein JI. Rare histological patterns of prostatic ductal adenocarcinoma. Pathology 2010;42: 319-324.

49 Srigley JR, Delahunt B, Evans AJ. Therapy-associated effects in the prostate gland. Histopathology 2012;60:153-165. 